\title{
Profound Thrombocytopenia after Treatment with Tirofiban; On-Pump Surgery for Acute Aortic Dissection and Coronary Bypass Surgery
}

Beckers Stefan ${ }^{1 *}$, Remory Isabel ${ }^{1}$, Van Der Meeren Sam², Bougie Daniel ${ }^{3}$, Demanet $\mathbf{C}^{2}$, Jochmans $\mathrm{K}^{2}$ and Poelaert Jan ${ }^{1}$

${ }^{1}$ Department of Anesthesiology and Perioperative Medicine, Universitair Ziekenhuis, Brussel, Belgium

${ }^{2}$ Department of Hematology, Universitair Ziekenhuis Brussel, Belgium

${ }^{3}$ Blood Center of Southeastern Wisconsin, Milwaukee, WI, USA

\begin{abstract}
We describe the case of a 69-year-old man who developed glycoprotein IIb/Illa receptor (GPIIb/Illa) antagonist associated thrombocytopenia and the successful management during urgent aortic dissection and coronary artery bypass grafting. A flow cytometric assay was developed to confirm the diagnosis of GPIlb/llla-antagonist induced thrombocytopenia. This case was further complicated by the concomitant presence of heparine/PF4 platelet antibodies, but was proven not to be the causal factor of the thrombocytopenia.
\end{abstract}

Keywords: Thrombocytopenia; IIb/IIIa glycoprotein inhibitors; Aortic dissection

\section{Introduction}

Problems related to the use of glycoprotein IIb/IIIa(GPIIb/IIIa) receptor antagonists, such as abciximab, eptifibatide and tirofiban, are not infrequent [1] and could have a major impact on the clinical course of an often critically ill patient. We describe a patient in whom severe thrombocytopenia was diagnosed, although the cause was initially unclear. Both heparin-induced thrombocytopenia and GPIIb/IIIa antagonist related antibodies obscured the picture, along with other possible causes of thrombocytopenia.

\section{Case Description}

A 69-year old male was transferred from a peripheral hospital to our University Hospital with suspicion of an inferoposterolateral infarction. He had a medical history involving arterial hypertension and a myocardial infarction, nineteen years ago. No allergy was known or any personal or familial bleeding history. Initially he was treated with acetylsalicylic acid, nadroparin, clopidogrel and tirofiban. The patient underwent a coronary angiography, revealing an occlusion of the left main artery and a $50 \%$ occlusion of the right coronary artery.

Furthermore, the aortography demonstrated an aortic dissection type I from the sinus of Valsalva to the right brachiocephalic trunk. A transthoracic echo-Doppler showed a massive aortic regurgitation, including a hypertrophic and hyperdynamic left ventricle. In addition aortic valve sclerosis and annular calcification was present.

The patient was scheduled for urgent coronary artery bypass grafting, and, therefore, could not consent with the treatment. A first preoperative blood count showed a dramatic fall of platelets to 3000/ $\mathrm{mm}^{3}$. Before the intervention, pseudo-thrombocytopenia was excluded based on the absence of aggregates in the platelets histogram and the exclusion of platelet clumps in the microscopic blood smear.

Although the patient remained hemodynamically stable, retrosternal pain aggravated, urging surgery despite this low number of platelets (Table 1). At the induction, a transfusion of one unit of platelets was administered in conjunction with methylprednisolon $0.5 \mathrm{mg} / \mathrm{kg}$ intravenously; moreover, nadroparin (low molecular weight heparin) and tirofiban treatment as possible causes of the thrombocytopenia were interrupted.

The surgeon reconstructed the ascending aorta with prosthesis, and put a venous bypass on both left anterior descending and right descending posterior coronary artery. An aortic valve plasty was completed. The patient was weaned from cardiopulmonary bypass and hemodynamically stabilized with an intra-aortic balloon pump, dobutamine (5 microgram/kg.min) and norepinephrin (150 ng/kg.min). The postoperative course was complicated with the development of a right bronchopneumonia, which was treated with amoxicillin. The platelet count remained normal without need for further transfusion and the patient was dismissed from the hospital 14 days postoperatively.

Blood samples, taken before cardiopulmonary bypass, were analyzed to elucidate the cause of the acute thrombocytopenia. Flow cytometric detection of tirofiban-dependent antibodies was performed in our hospital [1]. In brief, platelet-rich plasma was isolated from acid citrate dextrose anticoagulated blood, procured from normal group $\mathrm{O}$-donors. Platelets were further isolated by centrifugation in the presence of PGE1; dissolved in tyrodes-HEPES buffer and used immediately. $15 \mu$ platelets $\left(3 \times 10^{6}\right.$ till $\left.7 \times 10^{6}\right)$ were incubated with ten microliters of the patients' serum in $25 \mu$ l tyrodeshepes buffer in the presence or absence of $4 \mu \mathrm{g} / \mathrm{ml}$ tirofiban. After incubation for 1 hour at room temperature, the platelets were washed in buffer with or without $2 \mu \mathrm{g} / \mathrm{ml}$ drug. Platelet pellet was resuspended in $25 \mu \mathrm{l}$ tyrodes-HEPES buffer with or without $4 \mu \mathrm{g} / \mathrm{ml}$ tirofiban, and $25 \mu$ of FITC-labeled antihuman IgG diluted 1:40 was added.

Additionally RPE-labeled anti-CD41 was added to a final dilution of 1:20 to verify platelet identity on the flow cytometer. After a 45 incubation step, $300 \mu \mathrm{l}$ buffer $\pm 2 \mu \mathrm{g} / \mathrm{ml}$ tirofiban was added to the

${ }^{*}$ Corresponding author: Beckers S, Department of Anesthesiology and Perioperative Medicine, Universitair Ziekenhuis Brussel, Laarbeeklaan 101, 1090 Brussel, Belgium, E-mail: stefan.beckers@uzbrussel.be

Received January 15, 2013; Accepted January 27, 2013; Published January 29 2013

Citation: Stefan B, Isabel R, Sam VDM, Daniel B, Demanet C, et al. (2013) Profound Thrombocytopenia after Treatment with Tirofiban; On-Pump Surgery for Acute Aortic Dissection and Coronary Bypass Surgery. Cardiol Pharmacol 2: 105. doi:10.4172/2329-6607.1000105

Copyright: $\odot 2013$ Stefan B, et al. This is an open-access article distributed under the terms of the Creative Commons Attribution License, which permits unrestricted use, distribution, and reproduction in any medium, provided the original author and source are credited. 
samples and analyzed by flow cytometry Cytomics FC500, (Beckman Coulter). A positive reaction was defined as a shift in mean platelet fluorescence intensity compared to platelets processed identically, except for the presence of drug. An aliquot was sent to the Blood Research Institute of Blood Centre of Wisconsin for confirmation. These laboratory investigations have allowed us to specifically identify the presence of tirofiban-dependant antibodies (Figure 1). Because of the prior administration of both nadroparin and tirofiban, an antigencapture ELISA and a functional flow cytometric test were performed to investigate the possible presence and activity of IgG heparin/PF4 antibodies The former yielded positive results (OD 2.7, more positive as 0.5 ) while the functional flow cytometric test results were negative, which permitted us to exclude heparin-induced thrombocytopenia (HIT), as described previously [2].

After the intervention, the platelets number recovered quickly (Table 1). A year later, the patient was again admitted with de novo angina and underwent coronary angiography and subsequent CABG. Tirofiban was avoided and thrombocytopenia remained absent in the presence of heparin. The intervention occurred uneventful and the patient was dismissed after 12 days of hospitalization. After

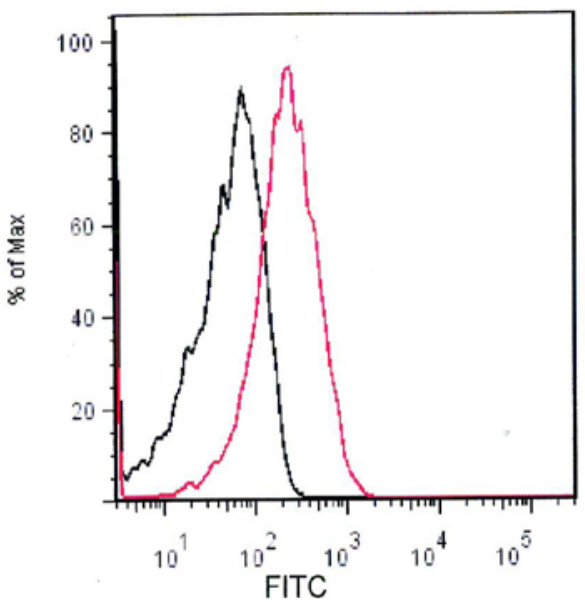

Figure 1: Tirofiban-dependant antibody test; Flowcytometry result showing a shift in fluorescence. From 16.43 to 218.01 (mean fluorescence intensity), indicating increase antibody binding in the presence of tirofiban. Result from Blood Centre of Wisconsin.

One parameter histogram with cell count on y-axis and anti-human IgG-FITC (fluorescein isothiocyanate conjugated detection antibody) measurement parameter on x-axis.

The black histogram represents control platelets with patient serum and the red histogram represents tirofiban treated platelets with patient serum. the procedure, the patient was informed and gave approval for the publication of the manuscript.

\section{Discussion}

GPIIb/IIIa antagonists (abciximab, eptifibatide, tirofiban) are widely used in the management of a variety of patients with acute coronary syndromes; they prevent platelet aggregation and thrombus formation, thereby improving outcomes of these patients [3]. Therapy with these agents may lead to bleeding complications and in rare cases to thrombocytopenia, challenging the perioperative management of these patients. In clinical trials with GPIIb/IIIa inhibitors, mild to severe decline of platelet counts has already been shown $[1,4,5]$. In particular, this decline has been demonstrated for abciximab in $\pm 2 \%$, often related to heterogeneous antibodies [6]. Tirofiban and eptifibatide show lower frequencies of associated thrombocytopenia [4,7].

This case demonstrates a profound acute thrombocytopenia after use of tirofiban, a GPIIb/IIIa inhibitor. Antibodies against tirofiban were undisputedly demonstrated in this patient. The differential diagnosis of the severe acute thrombocytopenia at initial presentation was pseudothrombocytopenia and two possible causes of drug-induced immune thrombocytopenia. Both heparin-induced thrombocytopenia (HIT) and tirofiban-related thrombocytopenia had to be excluded.

Pseudo-thrombocytopenia is defined as an in vitro clumping of platelets in blood samples anticoagulated with ethylenediaminetetraacetic acid (EDTA) [5,8]. In cases of pseudothrombocytopenia, there is neither in vivo thrombocytopenia nor enhanced aggregation. This artificial miscalculation of platelets was excluded after repeated low platelet counts in blood anticoagulated with citrate (Table 1) and because of the microscopical absence of platelet aggregates in blood smears. However, following Bizzaro [9], pseudothrombocytopenia can still occur in citrated blood samples. Ideally, to obviate this phenomenon, blood should be collected in ammonium oxalate and platelets counted in a Burker chamber.

HIT occurs due to the administration of the unfractionated or low molecular weight heparin and often leads to white thrombosis [10]. It typically presents at day 5-10 after first heparin administration, and the platelet count rarely falls below $20000 / \mathrm{mm}^{3}$ [11]. Because none of these criteria were met in our current case, the pre-test probability score for HIT was low.

However, cases of "fast-onset" HIT have been described and thus this potential cause had to be excluded [12]. IgG Heparin/PF4 antibodies were detected in the patient's serum, using an immunological HIT screening test (Zymutest HIA IgG, Hyphen Biomed). The activating potency of these antibodies was measured with a flow cytometric

\begin{tabular}{|c|c|c|c|c|c|c|c|c|c|c|c|}
\hline Time & $\begin{array}{c}20 / 11 \\
10: 30 \text { A.M. }\end{array}$ & $\begin{array}{c}\text { 20/11 } \\
14: 20 \text { P.M. }\end{array}$ & $\begin{array}{c}20 / 11 \\
\text { 15:30 P.M. }\end{array}$ & & $\begin{array}{c}20 / 11 \\
\text { 15:50 P.M. }\end{array}$ & $20 / 11$ & \multirow{2}{*}{ Start } & & \multirow{2}{*}{ ICU } & $\begin{array}{c}\text { 20/11 } \\
\text { 22:00 P.M. }\end{array}$ & $\begin{array}{c}21 / 11 \\
\text { 04:30 P.M. }\end{array}$ \\
\hline Platelet count $10^{3} / \mathrm{mm}^{3}$ & 265 & 3 & 3 (citr) & & 29 (citr) & 30 & & & & 152 & 132 \\
\hline PTT \% & 100 & 100 & & & & & \multirow{2}{*}{$\mathrm{N}$} & & \multirow{5}{*}{$\begin{array}{l}\mathrm{S} \\
\mathrm{T} \\
\mathrm{A} \\
\mathrm{R} \\
\mathrm{T}\end{array}$} & & \\
\hline APTT sec & 27 & 28.9 & & & & & & & & 35 & 34 \\
\hline Number of units of platelets & & & & 1 & & & \multirow{3}{*}{$\begin{array}{l}\mathrm{I} \\
\mathrm{E} \\
\mathrm{R} \\
\mathrm{V} \\
\mathrm{E} \\
\mathrm{N} \\
\mathrm{T} \\
\mathrm{I} \\
\mathrm{O} \\
\mathrm{N}\end{array}$} & \multirow[t]{2}{*}{6} & & & \\
\hline $\mathrm{Hb}$ g/dl & 15.2 & 13.8 & & & & & & & & 13.2 & 11.7 \\
\hline Htc $\%$ & 46 & 40.4 & & & & & & & & 37.9 & 34.5 \\
\hline
\end{tabular}

Table 1: Perioperative laboratory values after tirofiban and platelets administration. 
Citation: Stefan B, Isabel R, Sam VDM, Daniel B, Demanet C, et al. (2013) Profound Thrombocytopenia after Treatment with Tirofiban; On-Pump Surgery for Acute Aortic Dissection and Coronary Bypass Surgery. Cardiol Pharmacol 2: 105. doi:10.4172/2329-6607.1000105

Page 3 of 3

test, using CD62p (P-selectin) as a marker for platelet activation. This analysis was performed according to the method by Denys et al. [13]. Results were negative, thereby ruling out HIT. Moreover these antibodies can be excluded as cause of the thrombocytopenia from a clinical point of view: platelet levels restored to normal after withdrawal of tirofiban whereas the patient was kept on different drugs including heparin during further hospital stay.

Antibodies to heparin/PF4 complexes may exist in the absence of a clinical manifestation of HIT; in such case, the IgG heparin/PF4 antibodies present in the patient's serum are unable to activate the innate platelets. According to Warkentin and Greinacher, the variable platelet reactivity may correlate with the platelet Fc receptor-dependent reactivity and the existence of different polymorphisms of the receptor $[10,13]$. To elucidate this phenomenon, further effort was performed in our laboratory to identify the false positive results in the in vitro reaction or provide proof of the patient's platelet unresponsiveness.

The profound thrombocytopenia $\left(3000 / \mathrm{mm}^{3}\right)$ in our patient was provoked by the administration of tirofiban as shown by the flow cytometric detection of IgG solely in the presence of the drug tirofiban. The flow cytometry test (Figure 1) showed a shift in fluorescence in the presence of $4 \mu \mathrm{g} / \mathrm{ml}$, indicating increased antibody binding in the presence of tirofiban.

No shift in fluorescence was observed in the absence of tirofiban. In this case, the patient's plasma was unable to activate a pool of responsive donor platelets in response to added heparin, excluding HIT as the cause of thrombocytopenia. Therefore, it seemed unnecessary to perform further tests with the patient's innate platelets.

This case demonstrates once more the difficult differential diagnosis of acute thrombocytopenia. It also underlines the need of caution and rapid detection of drug-dependent antibodies with respect to GPIIb/ IIIa inhibitors, as these drugs will be used more frequently. The test is even more important in the light of the combined therapy with heparins, blurring the diagnosis in the presence of critical illness.

\section{References}

1. Bougie DW, Wilker PR, Wuitschick ED, Curtis BR, Malik M, et al. (2002) Acute thrombocytopenia after treatment with tirofiban or eptifibatide is associated with antibodies specific for ligand-occupied GPIIb/IIla. Blood 100: 2071-2076.

2. Tomer A (1997) A sensitive and specific functional flow cytometric assay for the diagnosis of heparin-induced thrombocytopenia. $\mathrm{Br} \mathrm{J}$ Haematol 98: 648-656.

3. Goto S, Tamura N, Li M, Handa M, Ikeda Y, et al. (2003) Different effects of various anti-GPIlb-IIla agents on shear-induced platelet activation and expression of procoagulant activity. J Thromb Haemost 1: 2022-2030.

4. Tcheng JE, Kereakes DJ, Lincoff AM, Georg BS, Kleiman NS, et al. (2001) Abciximab readministration: results of the ReoPro Readministration Registry Circulation 104: 870-875.

5. Wool RL, Coleman TA, Hamill RL (2002) Abciximab-associated pseudothrombocytopenia. Am J Med 113: 697-698.

6. Lajus S, Clofent-Sanchez G, Jais C, Coste P, Nurden P, et al. (2010) Thrombocytopenia after abciximab use results from different mechanisms. Thromb Haemost 103: 651-661.

7. Dasgupta H, Blankenship JC, Wood GC, Frey CM, Demko SL, et al. (2000) Thrombocytopenia complicating treatment with intravenous glycoprotein Ilb/IIla receptor inhibitors: a pooled analysis. Am Heart J 140: 206-211.

8. Dalamangas L, Slaughter T (1998) Ethylenediaminetetraacetic acid-dependant pseudothrombocytopenia in a cardiac surgical patient. Anesthesia Analgesia 86: $1210-1211$

9. Bizzaro N (1995) EDTA-dependant pseudothrombocytopenia: a clinical and epidemiological study of 112 cases, with 10-year follow-up. Am J Hematol 50 103-109.

10. Warkentin TE, Greinacher A (2004) Heparin-induced thrombocytopenia: recognition, treatment, and prevention: the Seventh ACCP Conference on Antithrombotic and Thrombolytic Therapy. Chest 126: 311S-337S.

11. Warkentin TE, Greinacher A (2009) Heparin-induced anaphylactic and anaphylactoid reactions: two distinct but overlapping syndromes. Expert Opin Drug Saf 8: 129-144

12. Kam PC, Egan MK (2002) Platelet Glycoprotein Ilb/Illa antagonists: pharmacology and clinical developments. Anesthesiology 96: 1237-1249.

13. Denys B, Stove V, Philippe J, Devreese K (2008) A clinical-laboratory approach contributing to a rapid and reliable diagnosis of heparin-induced thrombocytopenia. Thromb Res 123: 137-145. 